\title{
Future directions in kaonic atom physics
}

\author{
E. Friedman 1 ,* \\ ${ }^{1}$ Racah Institute of Physics, The Hebrew University, Jerusalem 91904, Israel
}

(Dated: August 3, 2018)

\begin{abstract}
Recent progress and open problems in kaonic atom physics are presented. A connection between phenomenological deep potentials and the underlying $K^{-} N$ interaction is established as well as the need for a theory for multinucleon absorption of kaons. $K^{-}$absorption at rest to specific $\Lambda$ hypernuclei states is briefly discussed.

PACS numbers: $13.75 . \mathrm{Jz}, 25.80 . \mathrm{Nv}, 36.10 .-\mathrm{k}$

Keywords: $K^{-} N$ interaction,kaonic atoms
\end{abstract}

\section{INTRODUCTION AND BACKGROUND}

Attempting to foresee directions of further research into the field of kaonic atoms one naturally focuses on open problems on the one hand and on recent progress on the other. All the data on strong interaction effects in mediumweight and heavy kaonic atoms come from experiments in the 1970's [1], using solid targets and a single GeLi X-ray detector. Analyses of the data show good consistency between the various experiments, and sub-sets of the data do not lead, in general, to conclusions which differ from what is found from analyses of the whole set of data. A new generation of experiments characterized by the use of many detectors and of tracking facilities for reducing background [2] and with excellent timing capabilities [3] addressed 'puzzles' with kaonic atoms of hydrogen and ${ }^{4} \mathrm{He}$.

Starting with medium-weight and heavy elements, a long standing problem with these kaonic atoms has been the question of the depth of the real optical potential. Deep potentials, in the range of $\operatorname{Re} V_{K^{-}}\left(\rho_{0}\right) \sim-(150-200) \mathrm{MeV}$ are obtained in comprehensive global fits to $K^{-}$-atom strong-interaction shift and width data by introducing empirical density dependent (DD) effective $K^{-} N$ amplitudes [4]. In contrast, considerably shallower potentials, in the range of $\operatorname{Re} V_{K^{-}}\left(\rho_{0}\right) \sim-(40-60) \mathrm{MeV}$, are obtained for zero kinetic-energy kaons when threshold chiral scattering amplitudes are used and self energy (SE) contributions are included in the in-medium corrections [5]. The large difference between the two results has been known as the 'deep vs. shallow puzzle' and is of interest not only because of the credibility of the two approaches but also because the depth of the potential may have far-reaching consequences for the possible existence of kaon-nucleon clusters. Very recently [6, 7] this problem was solved by using in-medium sub-threshold $K^{-} N$ scattering amplitudes which reproduced the empirical characterization of deep potentials [4], namely, that the real potentials are compressed relative to the nuclear charge distribution. These results demonstrated the need to supplement the theory with multi-nucleon absorption terms.

For the very light kaonic atoms new experiments [2, 3] removed the long-standing puzzle of kaonic hydrogen where now the results are fully consistent with the rest of the available data on antikaon-nucleon interactions near threshold.

*Electronic address: elifried@cc.huji.ac.il 


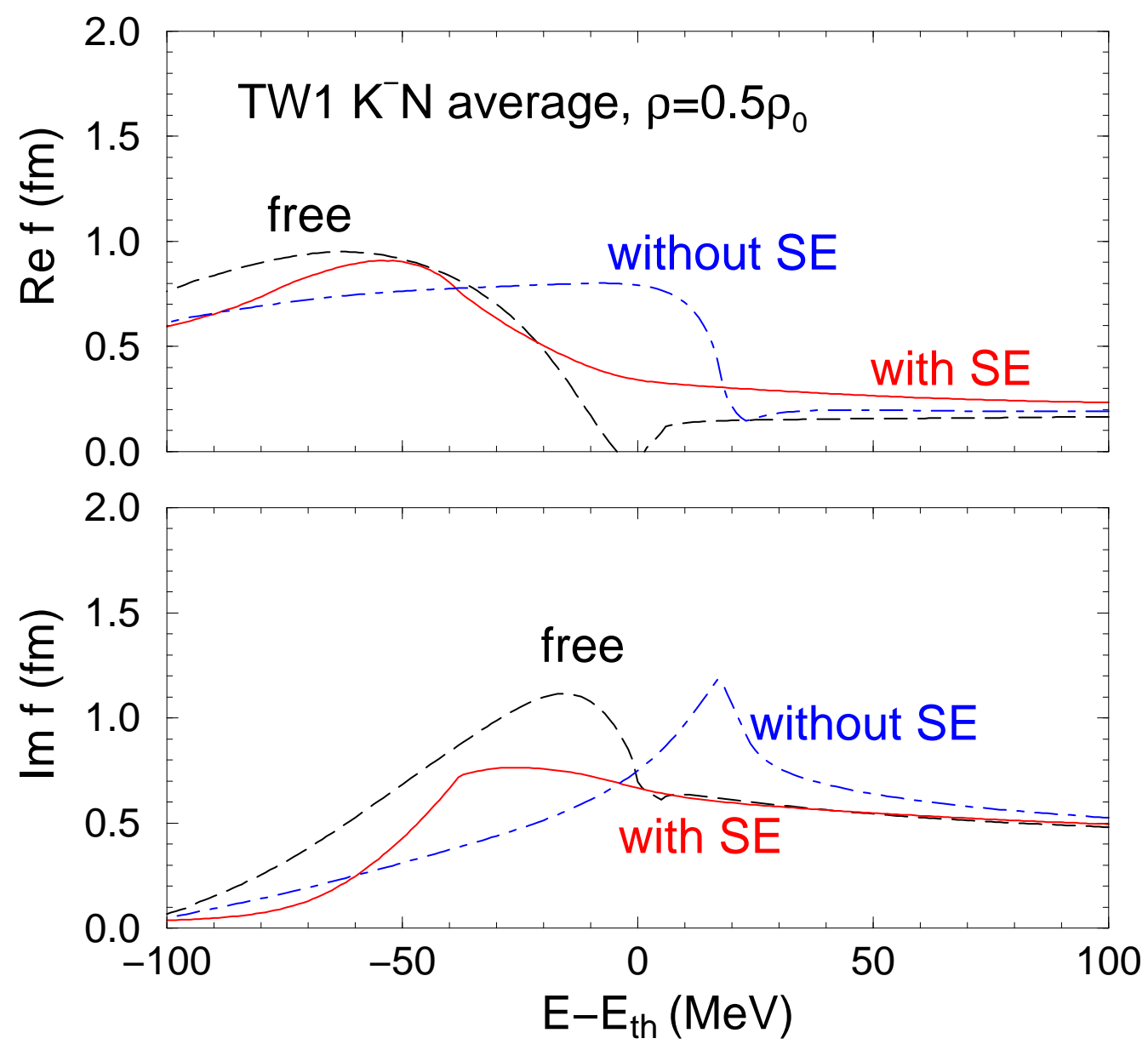

FIG. 1: Examples for average scattering amplitudes for a leading-order Tomozawa-Weinberg formulation. Dashed curves: free space, dot-dashed: Pauli blocked amplitude (without SE) at $\rho=0.5 \rho_{0}$, solid curves: including meson and baryon self energies (with SE) at $\rho=0.5 \rho_{0}$.

For ${ }^{4} \mathrm{He}$ the new results [8] removed the conflict of more than an order of magnitude between any credible theory and experiment.

The future directions outlined below result from this background.

\section{SUB-THRESHOLD AMPLITUDES}

Introducing empirical density dependent scattering amplitudes into global optical-model fits to data over the whole of the periodic table, deep real potentials were obtained that produced superior fits compared to fixed-amplitude approaches [4]. Another feature of these fits was a 'compression' of the real potential relative to the nuclear charge distribution. This result means that an underlying antikaon-nucleon amplitude must increase with the nuclear density such that it overshadows any finite-range effect that might be present. This is a general result, independent of details. We therefore focus attention on the density dependence of the $K^{-} N$ scattering amplitude, where its energy dependence near threshold is strongly affected by the proximity of the $\Lambda 1405$ resonance. Figure 1 shows chirally leading-order Tomozawa-Weinberg $K^{-} N$ scattering amplitudes marked TW1 solution, in free space and in symmetric 


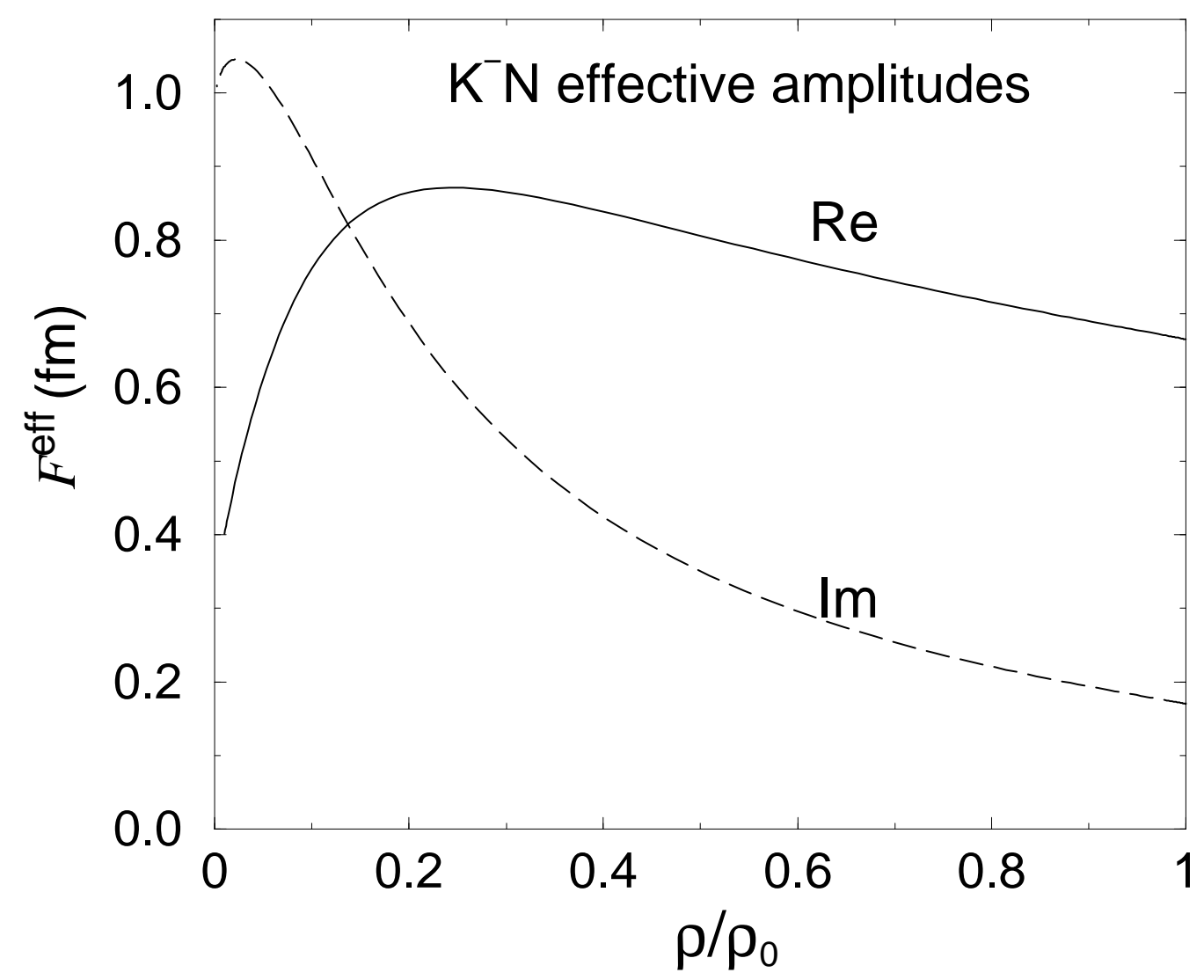

FIG. 2: Effective amplitudes for $K^{-} \mathrm{Ni}$ interaction.

nuclear medium for half the nuclear density, where medium corrections with self-energy and without are included, see refs. [6, 7] for details.

The in-medium amplitudes are defined by the Mandelstam parameter $s=\left(E_{K^{-}}+E_{N}\right)^{2}-\left(\vec{p}_{K^{-}}^{c m}+\vec{p}_{N^{\prime}}^{c m}\right)^{2}$ where the momenta are those implied by a kaon bound in the atom and by a nucleon which is part of the nucleus. Averaging over directions, using the Fermi gas model for the nucleus and taking the local kinetic energy for the kaon, one ends up with

$$
\sqrt{s} \approx E_{t h}-B_{N}-\xi_{N} B_{K}-15.1\left(\rho / \rho_{0}\right)^{2 / 3}+\xi_{K}\left(\operatorname{Re} V_{\mathrm{opt}}+V_{c}\right) .(\mathrm{MeV})
$$

in obvious notation, with $B_{N}$ an average binding energy for a nucleon and $\xi_{N}=m_{N} /\left(m_{N}+m_{K}\right), \quad \xi_{K}=m_{K} /\left(m_{N}+\right.$ $\left.m_{K}\right), \quad E_{t h}=m_{K}+m_{N}$. We note that the potential is proportional to the amplitude, but the amplitude depends on the potential through the argument $\sqrt{s}$. Therefore a self consistent iterative procedure was applied at every radial point for each nucleus in the data. That transforms the energy dependence into density dependence.

Figure2 2 shows an example for the density dependence of the scattering amplitude obtained for the $K^{-} N$ interaction in Ni. Near the nuclear surface the real part increases with density whereas the imaginary part decreases, in line with the compression and inflation of the real and imaginary potentials, respectively, observed in ref. [4]. The small values of the imaginary part at large densities is due to the sharp drop when energies go well below threshold, fig 1, towards the $\pi \Sigma$ threshold at $E-E_{t h} \simeq-100 \mathrm{MeV}$. 

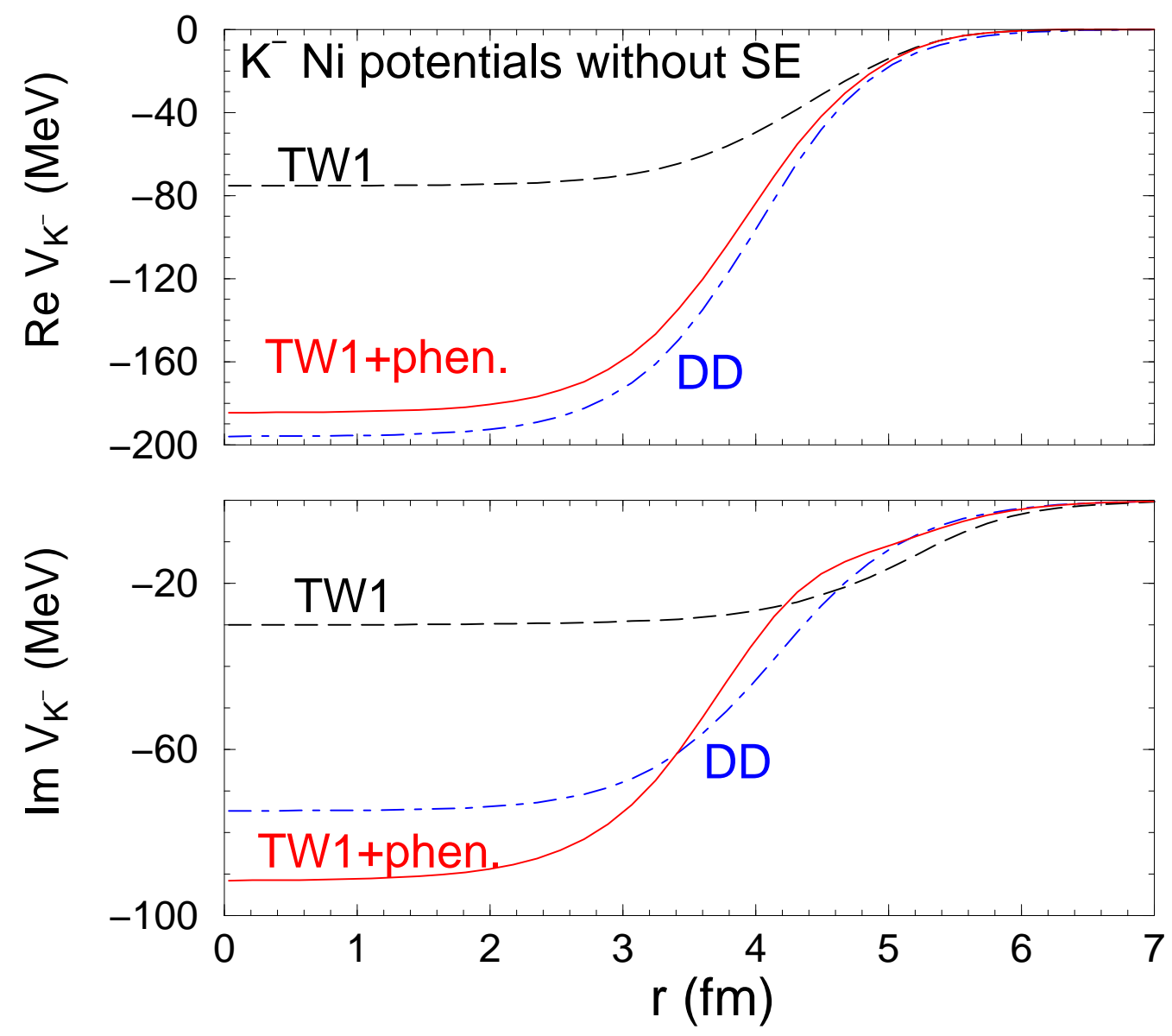

FIG. 3: $K^{-}$nuclear potentials for $K^{-}$atoms of Ni.

\section{KAONIC ATOM POTENTIALS}

\section{A. Medium-weight and heavy nuclei}

Comparisons between data and predictions made with optical potentials based on the above model lead to inadequate agreement with experiment, with $\chi^{2}$ per point of 10. Adding a phenomenological potential with four adjustable parameters (for 65 data points) leads to $\chi^{2}$ per point of 2 . This phenomenological term is dominated by $\rho^{2}$ dependence, suggesting indeed two-nucleon absorption which is not included in the present $K^{-} N$ amplitudes. A real dispersive term is to be expected.

Figure 3 shows examples for the $K^{-} \mathrm{Ni}$ potential. The real potential based on the TW1 amplitudes 7] is $80 \mathrm{MeV}$ deep compared to 40-50 MeV deep in ref. [5]. The cause for the latter is that ref. [5] used the amplitudes at threshold whereas figure 1 shows that at threshold the real part of the $\mathrm{SE}$ version is $\approx 50 \%$ of typical subthreshold values. For the full nuclear density the corresponding energy is as low as $50 \mathrm{MeV}$ below threshold. Also shown is the best fit potential when a phenomenological term is added, and for reference, also the purely empirical DD potential. It is tempting to reject the phenomenological additional term as being unacceptably large. However, the values at the nuclear center are not the meaningful quantities as kaonic atoms are sensitive mostly to the potential close to the nuclear surface [1]. 
TABLE I: Shifts and widths for the $2 p$ state in ${ }^{3,4} \mathrm{He}$.

\begin{tabular}{lll}
\hline & ${ }^{3} \mathrm{He}^{4} \mathrm{He}$ \\
shift $(\mathrm{eV})$ & 0.3 & -0.2 \\
width $(\mathrm{eV})$ & 2.1 & 1.6 \\
\hline
\end{tabular}

Considering that strong interaction effects in kaonic atoms are dominated by absorption, we note that the shape of the imaginary potential in the surface region $(\approx 4 \mathrm{fm}$ for $\mathrm{Ni})$ is modified by the phenomenological term by $\pm 30 \%$, close to estimates of multi-nucleon absorptions obtained from old emulsion and bubble-chamber experiments 9]. Replacing the additional phenomenological term by a multinucleon extension of the present 'microscopic' approach is a high priority line of research.

\section{B. Light nuclei}

Formation rates of hypernuclear states following the ${ }^{A} Z\left(K_{\text {stop }}^{-}, \pi^{-}\right)_{\Lambda}^{A} Z$ reaction have been another probe for studying the interaction of $K^{-}$mesons with nuclei at zero kinetic energy, where experimental results have been limited mostly to light $p$-shell nuclei. The analysis of these reactions is the other extreme compared to global analysis of kaonic atoms, by dealing separately not only with each nuclear species but also by handling explicitly specific kaonic atom states. Recent analysis of experimental results from FINUDA [10] showed 11] that with the sub-threshold approach the deep $K^{-}$-nucleus potential is favored. This conclusion is based on relative formation rates but the absolute scale of these is not reproduced by the calculations. Understanding the absolute scale is another direction of work in the field of kaonic atom physics.

Finally we turn to kaonic atoms of He. Table \ summarizes predictions made for ${ }^{3,4} \mathrm{He}$ with a global optical potential based on the TW1 amplitude plus a phenomenological term. Other variations such as the removal of the phenomenological term or the inclusion of a $p$-wave term change these values by up to $0.3 \mathrm{eV}$. However, future high precision experiment, well beyond the present capabilities, could bring interesting results.

\section{CONCLUSIONS}

Having established a connection between deep real optical potentials and underlying chiral-motivated $K^{-} N$ scattering amplitudes, the next step will be to implement a 'microscopic' multinucleon absorption terms to supplement the one-nucleon sub-threshold approach to kaonic atom potentials. Another near-future direction could be studies of the absolute scale of formation rates in stopped kaon absorption experiments where the current predictions underestimate experimental results by as much as a factor six. Refinements of global analyses by studies of different levels in a given kaonic atoms could bring interesting results. We note also that the role of a $p$-wave term in kaonic atoms has not been established [7]. Last but not least, any new high quality experiment will be most welcome. 


\section{Acknowledgments}

I wish to thank Aleš Cieplý, Avraham Gal, Daniel Gazda and Jiří Mareš who contributed much to the emerging sub-threshold approach.

[1] For reviews, C.J. Batty, E. Friedman, A.Gal, Strong interaction physics from hadronic atoms, Phys. Rep. 287, 385-445 (1997); E. Friedman and A. Gal, In-medium nuclear interactions of low-energy hadrons, Phys. Rep. 452, 89-153 (2007)

[2] M. Iwasaki et al., Observation of kaonic hydrogen $K_{\alpha}$ X Rays, Phys. Rev. Lett. 78, $3067-3069$ (1997)

[3] M. Bazzi et al., A new measurement of kaonic hydrogen X rays, Phys. Lett. B 704, 113-117 (2011)

[4] E. Friedman, A. Gal, C.J. Batty, Density-dependent $K^{-}$nuclear optical potential from kaonic atoms, Nucl. Phys. A579, 518-538 (1994)

[5] A. Ramos, E. Oset, Properties of (K)over-bar in the nuclear medium Nucl. Phys. A 671, 481-502 (2000)

[6] A. Cieplý et al., Chirally motivated $K^{-}$nuclear potentials, Phys. Lett. B 702, $402-407$ (2011)

[7] A. Cieplý et al., $K^{-}$nuclear potentials from in-medium chirally motivated models, Phys. Rev. C 84, 045206 (2011)

[8] S. Okada et al., Precision measurements of the $3 \mathrm{~d} \rightarrow 2 \mathrm{p}$ X-ray energy in kaonic ${ }^{4}$ He, Phys. Lett. B 653, 387-391 (2007)

[9] C. Vander Velde-Wilquet et al., Determination of branching fractions for K-mesons absorption at rest in carbon nuclei, Nuovo Cimento 39 A, 538-547 (1977); and references therein.

[10] M. Agnello et al., Hypernuclear spectroscopy with $K^{-}$at rest on ${ }^{7} \mathrm{Li},{ }^{9} \mathrm{Be},{ }^{13} \mathrm{C}$ and ${ }^{16} \mathrm{O}$, Phys. Lett. B 698, 219-225 (2011)

[11] A. Cieplý et al., Constraints on the threshold $K^{-}$nuclear potential from FINUDA ${ }^{A} Z\left(K_{s t o p}^{-}, \pi^{-}\right)_{\Lambda}^{A} Z$ spectra, Phys. Lett. B 698, 226-230 (2011) 